\title{
Malnutrition and Associated Factors in Admitted adult Surgical Patients in Eastern Ethiopia
}

\section{Zelalem Teklemariam}

Haramaya University

Fitsum Weldegebreal

Haramaya University

Habtamu Mitiku ( $\sim$ habtemit@gmail.com )

Haramaya University

\section{Research Article}

Keywords: malnutrition /under nutrition, surgical patients, eastern Ethiopia

Posted Date: December 18th, 2020

DOl: https://doi.org/10.21203/rs.3.rs-125072/v1

License: (c) (i) This work is licensed under a Creative Commons Attribution 4.0 International License. Read Full License 


\section{Abstract}

Background: Malnutrition in surgical patients is found to be associated with reduced wound healing, increased complication rates, length of hospital stay, mortality, and healthcare costs than normally nourished patients. There are higher magnitude was reported from surgical patients in different studies abroad. However, there is no study conducted in eastern Ethiopia. Therefore, this study was aimed to assess magnitude of malnutrition and associated factors among adult surgical patients.

Methods: Hospital based cross sectional study was conducted among 398 consecutively admitted surgical patients in Jugel and Hiwot Fana Specialized University Hospital from December 20, 2018 to November, 2019.Data was collected using pre tested structured questionnaire. Anthropometric measurements and dietary diversity assessment were made at admission and discharge of the patient. Capillary blood sample was collected for hemoglobin measurement. Data were analyzed using SPSS 20 software.

Result: A total87(21.9\%) and 99(24.9\%) study participants were underweight at admission and discharge. Being male (AOR=0.40; 95Cl: 0.21, 0.75), age groups $18-40$ years (AOR=0.22; $95 \mathrm{Cl}$ : 0.08, 0.62) and 41-60 years (AOR= $0.18 ; 95 \mathrm{Cl}: 0.06,0.53)$, urban residence $(A O R=0.38 ; 95 \%: 0.17,0.81)$ and Non anemic patients (AOR= $0.25 ; 95 \mathrm{Cl}: 0.12,0.48)$ were factors less likely to undernourished at admission. Being male

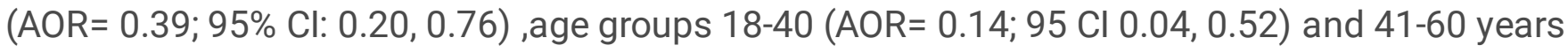
$(A O R=0.15 ; 95 \% \mathrm{Cl}: 0.04,0.52)$ urban residents $(A O R=0.26 ; 95 \mathrm{Cl}: 0.11,0.57)$, those with elective type of surgery (AOR $=0.34 ; 95 \% \mathrm{Cl}: 0.14,0.82$ ) less likely, participants with hospital stay less than 5 days $(A O R=0.14 ; 95 \mathrm{Cl} 0.02,0.69)$ were factors less likely to undernourished at discharge.

Conclusion: malnutrition /under nutrition is highly prevalent among surgical patients. Sex, age, residence and anemia were common factors associated with under nutrition at admission and discharge. In addition, type of current surgery and length of hospital stay were identified factors at time discharge. Therefore, the hospital staff should apply nutritional assessment and nutritional counseling /support to surgically admitted patients considering the identified factors.

\section{Introduction}

Malnutrition is rampant around the world which is a burden on patients and health care facilities. Studies reported that up to $40 \%$ of patients are malnourished at the time of their admission and the majority of them continued to be nutritionally depleted throughout their hospital course. Surgery related causes of malnutrition are due hyper catabolism, postoperative fasting, fistula, malabsorption syndrome, and intestinal obstruction (1-4).

Malnourished patients have higher morbidity and mortality rates than normally nourished patients, with longer hospital admissions and increased health care cost. In a study conducted in Singapore found that magnitude malnutrition at admission was 29\%; malnourished patients had longer hospital stays (6.9 \pm 7.3 days vs. $4.6 \pm 5.6$ days) and was more likely to be readmitted within 15 days (5). In another study 
report from German found a $27 \%$ rate of malnutrition among hospitalized patients. Malnourished patients having a LOS $43 \%$ longer than well-nourished patients (6).

Malnutrition is also found additionally associated with reduced wound healing and increased complication rates. Lack of appropriate nutritional support during hospitalization may worsen patients' nutritional status and increases risk for infection, organ failure and suboptimal response to regular medical treatment. (7).

Nutritional status at hospital admitted patients compounded by primary malnutrition mainly reflecting poor social-economic condition and secondary malnutrition reflecting usually the impact of degenerative and chronic diseases (2).

The treatment of malnutrition first requires identification of the patient as malnourished by means of nutrition screening or other type of assessment tool at admission. This procedure should be made compulsory by a health care team (8).

Magnitude of malnutrition among hospital admitted patients in Ethiopia was not widely well known and neglected. In one study conducted in Ethiopia among adult hospitalized patients the prevalence of malnutrition was $55.6 \%$ (9). The issue malnutrition among admitted patients is well studied other parts of the world $(3,10,11)$. However, there is no published data in the current study area. Therefore, this study tried to assess magnitude malnutrition and associated risk factors among Surgical Patients in public hospitals in eastern Ethiopia.

\section{Methods And Materials}

\section{Study area, design and period}

Harar town is the capital city of Harari National Regional state, which is one of the regional states of Federal Democratic Republic of Ethiopia and located at East part of Ethiopia at distance of $515 \mathrm{~km}$ from Addis Ababa. It is one of the most popular historical towns in the eastern part of Ethiopia. The town has a projected total population of 203,438 in 2010. The region has 8 worades and 36 kebeles (the lowest administrative structure). There are three governmental hospitals ( 2 public and 1 police hospital), two private hospitals and four health centers in the town (Harari region 2010.This cross-sectional study was conducted in the two public hospitals in the region i.e. Hiwot Fana Special University Hospital and Jugel Hospital, from December 20, 2018 to November, 2019.

\section{Population}

All admitted patients older than 18 years old of age in HiwotFana Specialized University Hospital and Jugel Hospital during the study period were source population. All surgical patients older than age of 18 years admitted to surgical ward and stayed in the ward for at least 24 hours were the study population. Because it is difficult to look how admission affects nutritional status patients with less than 24 hours admission. Patient having difficulty of communication and understanding, severe deformity, 
edematous patients, pregnant women, difficult to speak (on coma), who were not conscious, those who performed minor surgery and discharged before 24 hours, with recent surgery and re admitted again for another surgery (as these patients might have high malnutrition associated with previous surgery) were excluded from this study.

\section{Sample size and Sampling techniques}

Sample size was calculated using EPINFO version -software considering assumptions of power of $80 \%$, ratio unexposed: Exposed of 1:1; the prevalence of malnutrition among not read and write and literate was $61.5 \%$ and $48.5 \%$, respectively from study conducted among adult hospitalized patients at Amhara National Regional State, Ethiopia (9). The final sample for this study was 390 plus $5 \%$ non-response rate 410. The final Sample size was allocated proportionally based on average number of patients admitted to surgical ward in each hospital per month. Then all consecutive eligible patients were included from each hospital until the final sample size reached. Hiwot Fana specialized university hospital has 70 /month surgical admission with allocated sample size of 302; while Jugel Hospital has 25/month surgical admission with allocated sample of 108.

\section{Data collection methods}

Data were collected by the following methods

A. Face to face interview: was made by trained first degree in nursing as data collectors using pre-tested structured questionnaire which is prepared by reviewing related literatures $(9,12)$. The questionnaire was used to collected data on socio-demographic information such as age, occupation, education, income, type of transport and distance to hospital. In addition, clinical information from patient records such as current type of disease diagnosed and comorbidity, duration of current disease, previous history of surgery, complications after surgery and type of surgery was reviewed. After completion of the above data collection process during admission, study participants were followed till discharged from the hospital.

B. Nutritional status assessments: were conducted at the time admission and discharge. Weight (W) patients was measured using a scale with a maximum capacity of $150 \mathrm{~kg}$ and accuracy of $0.1 \mathrm{~kg}$. Weighing scale was checked for correctness after each patient measured. Height $(\mathrm{H})$ was measured in standing position in stadiometer but for those confounded to bed, demi-span (outstretched arms, forwarded palm from the base of the middle finger to the sterna notch), knee height and ulnar length were used (9). Waist circumference (WC) was measured on the median line between the costal border and the iliac crest at the end of exhalation. The above measurements were taken in duplicates and an average was used if different in measurement.

C. Dietary diversity assessment: was made by using standard dietary diversity questionnaire at admission and at time discharge (13).

D. Hemoglobin test: blood was collected through finger puncture and hemoglobin was measured using HemoCue hemoglobin analyzer (HemoCue® $\mathrm{Hb} 301$ ).

\section{Operational definition}


Length of hospital stay is measured in days, from the day of admission to hospital to the time of discharge or death.

\section{Malnutrition}

Malnutrition can apply to various states; undernutrition, over-nutrition or deficiencies of specific nutrients. In this study the term malnutrition refers to undernutrition.

\section{Data analysis}

Data was coded and entered using Epi Data software version 3.1 and transferred to SPSS version 20 software packages for analysis. A descriptive analysis of the patients was done by calculating mean, standard deviation and proportion of the studied variables. Dichotomous variables were expressed as percentages. The chi-square test was used to compare the proportions. The Student t-test was used to compare the mean of weight gain or loss at hospital admission and at discharge. Bivariate and multivariate logistic regression analysis was performed to assess factors associated with nutritional status at time of admission and discharge. Variables with $p$ value less than 0.25 were considered for multivariate analysis. Those variables with $p$ value less than 0.05 in multivariate analysis were considered as significantly associated factor.

Body Mass Index (BMI) $\left(\mathrm{kg} / \mathrm{m}^{2}\right)$ was calculated by dividing the weight by the square of the height $\left(\mathrm{kg} / \mathrm{m}^{2}\right)$. It was classified according to the World Health Organization criteria (WHO) (14) for adults, BMI < $18.5 \mathrm{~kg} / \mathrm{m} 2$ = Underweight; $18.5 \mathrm{~kg} / \mathrm{m} 2 \leq \mathrm{BMI} \leq 24.9 \mathrm{~kg} / \mathrm{m} 2=$ Normal; $25 \mathrm{~kg} / \mathrm{m} 2 \leq \mathrm{BMI} \leq 29.9 \mathrm{~kg} / \mathrm{m} 2=$ Overweight; $\mathrm{BMI} \geq 30 \mathrm{~kg} / \mathrm{m} 2$ = Obese. The BMl for elderly patients (60 years or older) was classified according to Lipschitz (15), BMI $\leq 22 \mathrm{~kg} / \mathrm{m} 2$ = underweight; $22 \mathrm{~kg} / \mathrm{m} 2<\mathrm{BMI}<27 \mathrm{~kg} / \mathrm{m} 2=$ Normal; $\mathrm{BMI} \geq$ $27 \mathrm{~kg} / \mathrm{m} 2$ = Overweight and $\mathrm{BMI} \geq 30 \mathrm{~kg} / \mathrm{m} 2=$ Obese. Waist circumference was transformed into dichotomous variables: higher than $94 \mathrm{~cm}$ for men or $80 \mathrm{~cm}$ for women was considered at risk according to International Diabetes Foundation (IDF) (16).

Individual Dietary Diversity Score was calculated as the sum of food groups consumed over 24 hours. By considering the mean individual dietary diversity score, participates with Individual dietary diversity Score above the mean were considered as good dietary diversity (diversified diet) or high and those below the mean Dietary Diversity Score were considered as low dietary diversity (undiversified diet) or low (13). Anemia was defined for non-pregnant women ( 15 years of age and above) and men ( 15 years of age and above) with hemoglobin value of $<11 \mathrm{~g} / \mathrm{dl}$ and $\leq 12 \mathrm{~g} / \mathrm{dl}$, respectively (17).

\section{Data quality}

Two days training was given for data collectors and first-degree holder public health officers supervisors how to collect and supervise. The questionnaire was first prepared in English then translated to local language and back translated to English to maintain the consistency of the questionnaire. Data collection instrument were pre-tested on $5 \%$ of the study participants at Dil Chora Hospital and appropriate modifications were made it. All the anthropometric measurements were taken using standard procedures 
and calibrated equipment by the data collector. Daily supervision, spot checking and reviewing completed questionnaire were conducted to follow for completeness and consistency of the data by the supervisors.

\section{Results}

\section{Socio-demographic characteristics}

A total of 398 study participants were included in this study with response rate of $97.1 \%$. Majority of them were from HiwotFana Specialized University Hospital (73.6\%). The mean age of the study participants was 35.6 (SD \pm 15.5 ) years and $279(70.1 \%)$ of them were in the range of 18-40 years. Most of the study participants were male (66.1\%) and rural resident (58\%). One hundred sixty-five (41.5\%) and $142(35.7 \%)$ of the participants were unable to read and write in educational status and farmers in their occupational status, respectively. Majority (88.4\%) of the participants earn $<3000$ birr per month (Table 1 ). 
Table 1

Socio-demographic characteristics of surgical patients admitted in Hiwot Fana Specialized University Hospital and Jugel, Hospital, 2019

\begin{tabular}{|c|c|c|c|}
\hline \multicolumn{2}{|l|}{ Characteristics } & \multirow{2}{*}{$\begin{array}{l}\text { Frequency } \\
279\end{array}$} & \multirow{2}{*}{$\begin{array}{l}\text { Percent } \\
70.1\end{array}$} \\
\hline Age & $18-40$ & & \\
\hline & $41-60$ & 85 & 21.4 \\
\hline & $>60$ & 34 & 8.5 \\
\hline \multirow[t]{2}{*}{ Residence } & Urban & 167 & 42.0 \\
\hline & Rural & 231 & 58.0 \\
\hline \multirow[t]{4}{*}{ Marital status } & Married & 256 & 64.3 \\
\hline & Divorced & 5 & 1.3 \\
\hline & Widowed & 12 & 3.0 \\
\hline & Unmarried & 125 & 31.4 \\
\hline \multirow[t]{5}{*}{ Educational status } & Not able to read and write & 165 & 41.5 \\
\hline & Able to read and write & 104 & 26.1 \\
\hline & Primary (Grade 1-8) & 65 & 16.3 \\
\hline & Secondary (9-12) & 37 & 9.3 \\
\hline & College and above & 27 & 6.8 \\
\hline \multirow[t]{7}{*}{ Occupational status } & Farmer & 142 & 35.7 \\
\hline & House wife & 60 & 15.1 \\
\hline & Government employed & 25 & 6.3 \\
\hline & Merchant & 60 & 15.1 \\
\hline & Daily laborer & 23 & 5.8 \\
\hline & Students & 65 & 16.3 \\
\hline & Other* & 23 & 5.8 \\
\hline \multirow[t]{3}{*}{ Monthly income $(n=364)$} & $<1000$ & 145 & 39.8 \\
\hline & $1000-3000$ & 177 & 48.6 \\
\hline & $3001-5000$ & 42 & 11.6 \\
\hline
\end{tabular}


Majority study participants $(72.6 \%)$ have with the current diseases of < 2 weeks duration. Only 30 (7.5\%) of participants had history surgery. A total of $215(54.0 \%)$ and $183(46 \%)$ were on emergency and elective surgery, respectively. Forty-three $(10.2 \%)$ of study participants had co-morbidities other than the current disease they admitted. Diabetes mellitus was the commonest co-morbidity followed by hypertension. Sixty-two (15.6\%) patients developed complications after surgery and surgical site wound infection was the commonest complication followed by sepsis. The median length of stay of study participants in the hospital was 9.3 days and majority $(77.1 \%)$ of them stayed $5-15$ days. The mean hemoglobin concentration was $12.7(\mathrm{SD} \pm 1.9) \mathrm{g} / \mathrm{dl}$. Most of the study participants were admitted due to digestive tract diseases (39.7\%) and trauma (29.1) (Table 2). A total of $392(98.5 \%)$ and $6(1.5 \%)$ of the study participant were healed and referred out at time discharge, respectively. 
Table 2

Clinical characteristics of surgical patients admitted in Hiwot Fana Specialized University Hospital and Jugel Hospital, 2019.

\begin{tabular}{|c|c|c|c|}
\hline \multicolumn{2}{|l|}{ Characteristics } & \multirow{2}{*}{$\begin{array}{l}\text { Frequency } \\
289\end{array}$} & \multirow{2}{*}{$\begin{array}{l}\text { Percent } \\
72.6\end{array}$} \\
\hline Duration of disease & $<2$ weeks & & \\
\hline & $\geq 2-4$ weeks & 109 & 27.4 \\
\hline \multirow[t]{2}{*}{ History of surgery } & Yes & 30 & 7.5 \\
\hline & No & 368 & 92.5 \\
\hline \multirow[t]{8}{*}{ Co-morbidity } & No-co-morbidity & 355 & 89.2 \\
\hline & Diabetes Mellitus & 19 & 4.8 \\
\hline & Hypertension & 13 & 3.3 \\
\hline & Hypothyroidism & 2 & 0.5 \\
\hline & Coagulopathy & 5 & 1.3 \\
\hline & Malaria & 1 & 0.3 \\
\hline & HIV & 1 & 0.3 \\
\hline & Psychoses & 2 & 0.5 \\
\hline \multirow[t]{5}{*}{ Complication after surgery } & Not at all & 336 & 84.4 \\
\hline & Sepsis & 18 & 4.5 \\
\hline & Pneumonia & 7 & 1.8 \\
\hline & Urinary tract infection & 4 & 1.0 \\
\hline & Wound Infection & 33 & 8.3 \\
\hline \multirow[t]{3}{*}{ Length of Hospital stay } & $<5$ days & 57 & 14.3 \\
\hline & 5-15 days & 307 & 77.1 \\
\hline & $>15$ days & 34 & 8.5 \\
\hline \multirow[t]{6}{*}{ Current diseases of patient admitted } & Digestive tract & 158 & 39.7 \\
\hline & Malignant neoplasm & 10 & 2.5 \\
\hline & Urological & 37 & 9.3 \\
\hline & Muscular & 26 & 6.5 \\
\hline & Trauma & 116 & 29.1 \\
\hline & Amputation & 10 & 2.5 \\
\hline
\end{tabular}




\begin{tabular}{|llll|}
\hline Characteristics & Frequency & Percent \\
\hline & Other * & 41 & 10.3 \\
\hline
\end{tabular}

\section{Support and dietary diversity}

About $64.8 \%$ (258/398) of the study participants reported that they got support from relative, friends, or neighbors during their admission. Majority $(90.7 \%)$ of them were obtained support from their relatives. Nutrition support (67.7\%) was the commonest type of support. Only $16.8 \%(31 / 184)$ of those study participants with elective surgery obtained nutritional support before two weeks of admission. About $43.5 \%$ (31/398) of those who did surgery obtained nutritional counseling support during hospital stay. Two hundred seventy-three (68.6\%) and 149 (37.4\%) of study participants had low dietary diversity score at admission and at discharge, respectively (Table 3 ). 
Table 3

Support and dietary diversity of admitted surgical patients in Hiwot Fana Specialized University Hospital and Jugel Hospital, 2019

\begin{tabular}{|c|c|c|c|}
\hline Characteristics & & Frequency & Percent \\
\hline \multirow[t]{3}{*}{ Who gave the support $(n=258)$} & Relative & 234 & 90.7 \\
\hline & Friends & 20 & 7.8 \\
\hline & Neighbors & 4 & 1.6 \\
\hline \multirow[t]{7}{*}{ Type of support got $(n=258)$} & Nutrition & 174 & 67.7 \\
\hline & Medication & 11 & 4.3 \\
\hline & Counseling & 5 & 1.9 \\
\hline & $\begin{array}{l}\text { Nutrition and } \\
\text { Medication }\end{array}$ & 18 & 7.0 \\
\hline & $\begin{array}{l}\text { Nutrition and } \\
\text { Counseling }\end{array}$ & 26 & 10.1 \\
\hline & $\begin{array}{l}\text { Nutrition, } \\
\text { Medication and } \\
\text { counseling }\end{array}$ & 20 & 7.8 \\
\hline & Money & 4 & 1.6 \\
\hline \multirow{2}{*}{$\begin{array}{l}\text { Nutritional counseling support before two weeks of } \\
\text { admission for those with elective surgery }(n=183)\end{array}$} & Yes & 30 & 16.4 \\
\hline & No & 153 & 83.6 \\
\hline \multirow{2}{*}{$\begin{array}{l}\text { Nutritional counseling support during hospital stay ( } \\
=398 \text { ) }\end{array}$} & Yes & 173 & 43.5 \\
\hline & No & 225 & 52.5 \\
\hline \multirow[t]{2}{*}{ Dietary diversity at admission } & Low & 273 & 68.6 \\
\hline & High & 125 & 31.4 \\
\hline \multirow[t]{2}{*}{ Dietary diversity at discharge } & Low & 149 & 37.4 \\
\hline & High & 249 & 62.6 \\
\hline
\end{tabular}

\section{Nutritional status at admission and discharge}

The mean weight of the study participants at admission and discharge was 57.7 (SD \pm 10.5 ) and 57.4 ( $S D \pm 10.6)$. The mean BMI of the study participants during admission and discharge was 21.1(SD \pm 3.3 ) and $21(\mathrm{SD} \pm 3.4)$, respectively (P-value < 0.20$)$. From 398 admitted patients $87(21.9 \%)(95 \% \mathrm{Cl}: 17.9,26.2)$ and 99 (24.9\%) (95\% Cl: 20.7,29.4) were underweight at admission and discharge, respectively. A total of 42 (10.6\%) (95\% Cl: 7.714 .0$)$ and $48(12.1 \%)(95 \% \mathrm{Cl}: 9.0,15.7)$ study participants had risk of chronic disease during admission and discharge based on waist circumference measurement respectively (Table 4). 
Table 4

Nutritional status of surgical patients admitted in Hiwot Fana Specialized University Hospital and Jugel Hospital 2019

\begin{tabular}{|c|c|c|c|c|}
\hline \multicolumn{2}{|l|}{ Nutritional status } & $\begin{array}{l}\text { At admission } \\
\text { No. (\%) }\end{array}$ & $\begin{array}{l}\text { At discharge } \\
\text { No. (\%) }\end{array}$ & $\begin{array}{l}P \text { - } \\
\text { value }\end{array}$ \\
\hline Mean weight & & $\begin{array}{l}57.7(\mathrm{SD} \pm \\
10.5)\end{array}$ & $\begin{array}{l}57.4(\mathrm{SD} \pm \\
10.6)\end{array}$ & 0.00 \\
\hline Mean BMI & & $\begin{array}{l}21.1(\mathrm{SD} \pm \\
3.3)\end{array}$ & $21(S D \pm 3.4)$ & 0.20 \\
\hline \multirow[t]{4}{*}{ BMI } & Under weight & $87(21.9)$ & $99(24.9)$ & 0.31 \\
\hline & Normal weight & $278(69.8)$ & $264(66.3)$ & 0.29 \\
\hline & Over weight & $28(7.0)$ & $28(7.0)$ & 1.00 \\
\hline & Obese & $5(1.3)$ & $7(1.8)$ & 0.56 \\
\hline \multirow[t]{2}{*}{$\begin{array}{l}\text { Waist } \\
\text { circumference }\end{array}$} & $\begin{array}{l}<94 \mathrm{~cm} \text { for males, }<80 \mathrm{~cm} \text { for } \\
\text { females }\end{array}$ & $356(89.4)$ & $350(87.9)$ & \multirow[t]{2}{*}{0.50} \\
\hline & $\begin{array}{l}\geq 94 \mathrm{~cm} \text { for males } \geq 80 \mathrm{~cm} \text { for } \\
\text { female }\end{array}$ & $42(10.6)$ & $48(12.1)$ & \\
\hline
\end{tabular}

\section{Factors associated with under nutrition at admission}

In bivariate analysis age, residence and anemia status were found to be significantly associated with under nutrition during admission $(P<0.05)$. However, in multivariate logistic regression analysis sex, age and residence were found independent predictors of under nutrition during admission.

Being male in sex were $60 \%(A O R=0.40 ; 95 \% \mathrm{Cl}: 0.21,0.75)$ less likely to be undernourished compare to female sex at admission. Age group $18-40$ years $78 \%(\mathrm{AOR}=0.22 ; 95 \% \mathrm{Cl}: 0.08,0.62)$ and $41-60$ years $82 \%(\mathrm{AOR}=0.18 ; 95 \% \mathrm{Cl}: 0.06,0.53)$ less likely to be undernourished compare to those patients $>60$ years of age. Patients from urban areas $72 \%$ (AOR $=0.38 ; 95 \%: 0.17,0.81$ ) less likely to be undernourished compare to those patients from rural areas (Table 5). 
Table 5

Factors associated with under nutrition at admission among surgical patients in Hiwot Fana Specialized University Hospital and Jugel Hospital $2019(\mathrm{~N}=365)$

\begin{tabular}{|c|c|c|c|c|c|}
\hline \multirow[t]{3}{*}{ Variables } & & \multicolumn{2}{|c|}{ Nutritional status } & \multirow{3}{*}{$\begin{array}{l}\mathrm{COR} \\
\text { at } 95 \% \mathrm{Cl}\end{array}$} & \multirow{3}{*}{$\begin{array}{l}\text { AOR } \\
\text { at } 95 \% \mathrm{Cl}\end{array}$} \\
\hline & & \multirow{2}{*}{$\begin{array}{l}\text { Under } \\
\text { nourished } \\
\text { No.(\%) }\end{array}$} & \multirow{2}{*}{$\begin{array}{l}\text { Not under } \\
\text { nourished } \\
\text { No.(\%) }\end{array}$} & & \\
\hline & & & & & \\
\hline \multirow[t]{2}{*}{ Hospitals } & HFSUH & $71(25.8)$ & $204(74.2)$ & $\begin{array}{l}1.61(0.88 \\
2.94)\end{array}$ & $\begin{array}{l}1.68(0.68 \\
4.12)\end{array}$ \\
\hline & Jugel & 16(17.8) & $74(82.2)$ & 1 & 1 \\
\hline \multirow[t]{3}{*}{ Age } & $18-40$ & $58(22.1)$ & 205(77.9) & $\begin{array}{l}0.26(012 \\
0.56)\end{array}$ & $\begin{array}{l}0.22(0.08 \\
0.62)\end{array}$ \\
\hline & $41-60$ & 13(18.3) & $58(81.7)$ & $\begin{array}{l}0.21(0.08 \\
0.53)\end{array}$ & $\begin{array}{l}0.18(0.06 \\
0.53)\end{array}$ \\
\hline & $>60$ & 16(51.6) & 15(48.4) & 1 & 1 \\
\hline \multirow[t]{2}{*}{ Sex } & Male & $54(22.5)$ & 186(77.5) & $\begin{array}{l}1.23(0.74 \\
2.03)\end{array}$ & $\begin{array}{l}0.40(0.21 \\
0.75)\end{array}$ \\
\hline & Female & $33(26.4)$ & 92(73.6) & 1 & 1 \\
\hline \multirow[t]{2}{*}{ Residence } & Urban & $17(12.0)$ & 125(88.0) & $\begin{array}{l}0.29(0.16 \\
0.53)\end{array}$ & $\begin{array}{l}0.38(0.17 \\
0.81)\end{array}$ \\
\hline & Rural & $70(31.4)$ & 153(68.6) & 1 & 1 \\
\hline \multirow[t]{3}{*}{$\begin{array}{l}\text { Educational } \\
\text { status }\end{array}$} & $\begin{array}{l}\text { Not able to read and } \\
\text { write }\end{array}$ & $43(28.1)$ & 110(71.9) & $\begin{array}{l}1.79(0.83 \\
3.88)\end{array}$ & $\begin{array}{l}0.65(0.22 \\
1.87)\end{array}$ \\
\hline & $\begin{array}{l}\text { Able to read and write } \\
\text { and primary }\end{array}$ & $34(21.8)$ & $122(78.2)$ & $\begin{array}{l}1.28(0.58 \\
2.80)\end{array}$ & $\begin{array}{l}0.85(0.32 \\
2.29)\end{array}$ \\
\hline & $\begin{array}{l}\text { High school and } \\
\text { above }\end{array}$ & $10(17.9)$ & $46(82.1)$ & 1 & 1 \\
\hline \multirow[t]{3}{*}{$\begin{array}{l}\text { Monthly } \\
\text { income }\end{array}$} & $<1000$ & $37(26.2)$ & 104(73.8) & $\begin{array}{l}1.38(0.55 \\
3.45)\end{array}$ & $1.87(0.58,6.07)$ \\
\hline & $1000-3000$ & $33(20.8)$ & $126(79.2)$ & $\begin{array}{l}1.01(0.40 \\
2.52)\end{array}$ & $\begin{array}{l}\text { 1.32(0.42, } \\
4.06)\end{array}$ \\
\hline & $3001-5000$ & $7(20.6)$ & $27(79.4)$ & 1 & 1 \\
\hline \multirow[t]{2}{*}{$\begin{array}{l}\text { Duration of } \\
\text { disease }\end{array}$} & $<2$ weeks & $57(21.3)$ & 211(78.7) & $\begin{array}{l}0.60(0.35 \\
1.01)\end{array}$ & $\begin{array}{l}0.85(0.43 \\
1.68)\end{array}$ \\
\hline & $>2-4$ weeks & $30(30.9)$ & $67(69.1)$ & 1 & 1 \\
\hline $\begin{array}{l}\text { History of } \\
\text { surgery }\end{array}$ & Yes & $7(33.3)$ & 14(66.7) & $\begin{array}{l}76.7(0.64 \\
4.22)\end{array}$ & $\begin{array}{l}\text { 1.63(0.45, } \\
5.86)\end{array}$ \\
\hline
\end{tabular}




\begin{tabular}{|llllll|} 
& No & $80(23.3)$ & $264(76.7)$ & 1 & 1 \\
\hline Co-morbidity & Yes & $9(25.0)$ & $27(75.0)$ & 1 & 1 \\
\cline { 2 - 6 } & No & $78(23.7)$ & $251(76.3)$ & $0.93(0.42$, & $0.98(0.31$, \\
& & & $2.06)$ & $3.11)$ \\
Anemic & Non anemic & $46(17.9)$ & $211(82.1)$ & $0.34(0.20$, & $0.25(0.12$, \\
status & & & & $0.57)$ & $0.48)$ \\
(n=360) & Anemic & $40(38.8)$ & $63(61.2)$ & 1 & 1 \\
\cline { 2 - 6 } & & & & 1 & 1 \\
\hline
\end{tabular}

\section{Factors associated with under nutrition at discharge}

In bivariate analysis the hospital where patients admitted, age, residence and length of hospital stay were found to be significantly associated with under nutrition during admission $(P<0.05)$. However, in multivariate logistic regression analysis sex, age, residence and type of current surgery and length of hospital stay were factors associated with under nutrition during discharge.

Males were $61 \%(A O R=0.39 ; 95 \% \mathrm{Cl}: 0.20,0.76)$ less likely to be undernourished compare to females. Age groups $18-40$ years were $86 \%(\mathrm{AOR}=0.14 ; 95 \% \mathrm{Cl} 0.04,0.52)$ and $41-60$ years were $85 \%(\mathrm{AOR}=$ $0.15 ; 95 \% \mathrm{Cl}: 0.04,0.52)$ less likely to be undernourished compare to those patients $>60$ years of age. Patients from urban areas were $74 \%(\mathrm{AOR}=0.26 ; 95 \% \mathrm{Cl}: 0.11,0.57)$ less likely to be undernourished compare to those from rural areas. Those patients who under gone elective type of surgery were $66 \%$ $(A O R=0.34 ; 95 \% \mathrm{Cl}: 0.14,0.82)$ less likely to be undernourished compare to those patients under gone emergency surgery. Those patients stayed in the hospital for $<5$ days were $86 \%$ (AOR $=0.14 ; 95 \% \mathrm{Cl} 0.02$, 0.69 ) less likely to be undernourished compare to those stayed more than 15 days. Those patients who are non-anemic were $78 \%$ less likely to be undernourished compare to anemic ( $\mathrm{AOR}=0.22 ; 95 \% \mathrm{Cl}: 0.10$, 0.45) (Table 6). 
Table 6

Factors associated with under nutrition at discharge among surgical patients admitted in Hiwot Fana Specialized University Hospital and Jugel Hospital 2019. ( $\mathrm{N}=363)$

\begin{tabular}{|c|c|c|c|c|c|}
\hline \multirow[t]{3}{*}{ Variables } & & \multicolumn{2}{|c|}{ Nutritional status } & \multirow{3}{*}{$\begin{array}{l}\text { COR } \\
95 \% \mathrm{Cl}\end{array}$} & \multirow{3}{*}{$\begin{array}{l}\text { AOR } \\
95 \% \mathrm{Cl}\end{array}$} \\
\hline & & \multirow{2}{*}{$\begin{array}{l}\text { Under } \\
\text { nourished } \\
\text { No. (\%) }\end{array}$} & \multirow{2}{*}{$\begin{array}{l}\text { Not under } \\
\text { nourished } \\
\text { No. (\%) }\end{array}$} & & \\
\hline & & & & & \\
\hline \multirow[t]{2}{*}{ Hospitals } & HFSUH & 83(30.3) & 191(69.7) & $1.98(1.08,3.61)$ & $2.56(0.71,9.18)$ \\
\hline & Jugel & 16(18.0) & $73(82.0)$ & 1 & 1 \\
\hline \multirow[t]{3}{*}{ Age } & $18-40$ & $66(25.3)$ & 195(74.7) & $0.24(0.11,0.52)$ & $0.14(0.04,0.52)$ \\
\hline & $41-60$ & $15(21.1)$ & $56(78.9)$ & $0.19(0.07,0.48)$ & $0.15(0.04,0.52)$ \\
\hline & $>60$ & $18(58.1)$ & $13(41.9)$ & 1 & 1 \\
\hline \multirow[t]{2}{*}{ Sex } & Male & $59(24.8)$ & $179(75.2)$ & $0.70(0.43,1.12)$ & $0.39(0.20,0.76)$ \\
\hline & Female & $40(32.0)$ & $85(68.0)$ & 1 & 1 \\
\hline \multirow[t]{2}{*}{ Residence } & Urban & 19(13.4) & $123(86.6)$ & $0.27(0.15,0.47)$ & $0.26(0.11,0.57)$ \\
\hline & Rural & $80(36.2)$ & $141(63.8)$ & 1 & 1 \\
\hline \multirow[t]{3}{*}{$\begin{array}{l}\text { Educational } \\
\text { status }\end{array}$} & $\begin{array}{l}\text { Not able to } \\
\text { read and write }\end{array}$ & $46(30.1)$ & 107(69.9) & $1.57(.76,3.25)$ & $0.49(0.16,1.46)$ \\
\hline & $\begin{array}{l}\text { Able to read } \\
\text { and write and } \\
\text { primary }\end{array}$ & $41(26.6)$ & $113(73.4)$ & $1.33(0.64,2.76)$ & $1.04(0.39,2.78)$ \\
\hline & $\begin{array}{l}\text { High school } \\
\text { and above }\end{array}$ & $12(21.4)$ & $44(78.6)$ & 1 & 1 \\
\hline \multirow{3}{*}{$\begin{array}{l}\text { Monthly } \\
\text { income }(n=364)\end{array}$} & $<1000$ & $44(32.1)$ & $93(67.9)$ & $1.80(0.73,4.46)$ & $2.17(0.59,7.92)$ \\
\hline & $1000-3000$ & $36(22.5)$ & $124(77.5)$ & $1.12(0.45,2.78)$ & $1.63(0.45,5.87)$ \\
\hline & $3001-5000$ & $7(20.6)$ & $27(79.4)$ & 1 & 1 \\
\hline \multirow{2}{*}{$\begin{array}{l}\text { Dietary diversity } \\
\text { at discharge }\end{array}$} & Low & $29(22.3)$ & 101(77.7) & $0.66(0.40,1.10)$ & $0.90(0.38,2.15)$ \\
\hline & High & $70(30.0)$ & 163(70.0) & 1 & 1 \\
\hline \multirow{2}{*}{$\begin{array}{l}\text { Duration of } \\
\text { disease }\end{array}$} & $<2$ weeks & $68(25.5)$ & 199(74.5) & $0.71(0.43,1.19)$ & $0.69(0.27,1.75)$ \\
\hline & $\geq 2$ weeks & $31(32.3)$ & $65(67.7)$ & 1 & 1 \\
\hline \multirow{2}{*}{$\begin{array}{l}\text { History of } \\
\text { surgery }\end{array}$} & Yes & $7(33.3)$ & $14(66.7)$ & $1.35(0.53,3.47)$ & $1.67(0.40,6.87)$ \\
\hline & No & $92(26.9)$ & $250(73.1)$ & 1 & 1 \\
\hline Type of current & Elective & $41(26.1)$ & 116(73.9) & $0.90(0.56,1.44)$ & $0.34(0.14,0.82)$ \\
\hline
\end{tabular}




\begin{tabular}{|c|c|c|c|c|c|}
\hline surge & Emergency & $58(28.2)$ & 148(71.8) & 1 & 1 \\
\hline \multirow[t]{2}{*}{ Co-morbidity } & Yes & $9(25.0)$ & $27(75.0)$ & 1 & 1 \\
\hline & No & $90(27.5)$ & $237(72.5)$ & $1.13(0.51,2.5)$ & $0.67(0.18,2.43)$ \\
\hline \multirow{2}{*}{$\begin{array}{l}\text { Complication } \\
\text { after surgery }\end{array}$} & Yes & $20(35.7)$ & $36(64.3)$ & 1 & 1 \\
\hline & No & $79(25.7)$ & $228(74.3)$ & $0.624(0.34,1.14)$ & $0.67(0.18,2.43)$ \\
\hline \multirow{3}{*}{$\begin{array}{l}\text { Length of } \\
\text { Hospital stay }\end{array}$} & $<5$ days & $7(12.7)$ & $48(87.3)$ & $0.14(0.05,0.42)$ & $0.14(0.02,0.69)$ \\
\hline & 5-15 days & $77(27.7)$ & $201(72.3)$ & $0.38(.179,0.82)$ & $0.46(0.14,1.50)$ \\
\hline & $>15$ days & $15(50.0)$ & $15(50.0)$ & 1 & 1 \\
\hline \multirow{2}{*}{$\begin{array}{l}\text { Nutritional } \\
\text { support during } \\
\text { hospital stay }\end{array}$} & Yes & $48(30.0)$ & 112(70.0) & $1.27(0.80,2.03)$ & $0.92(0.49,1.71)$ \\
\hline & No & $51(25.1)$ & 152(74.9) & 1 & 1 \\
\hline \multirow{2}{*}{$\begin{array}{l}\text { Category of } \\
\text { disease }\end{array}$} & Malignant & 18(51.4) & 17(48.6) & $3.22(1.58,6.55)$ & $1.95(0.73,5.22)$ \\
\hline & Non malignant & $81(24.7)$ & $247(75.3)$ & 1 & 1 \\
\hline \multirow{2}{*}{$\begin{array}{l}\text { Anemic status } \\
(\mathrm{n}=358)\end{array}$} & Non anemic & $56(22.0)$ & 199(78.0) & $0.40(0.25,0.66)$ & $\begin{array}{l}0.22(0.10 \\
0.45)\end{array}$ \\
\hline & Anemic & $42(40.8)$ & $61(59.2)$ & 1 & 1 \\
\hline
\end{tabular}

\section{Discussion}

The magnitude of underweight / under nutrition was increased $21.9-24.9 \%(p>0.05)$ and obesity from $5(1.3)$ to $7(1.8)$ and waist circumference of $\geq 94 \mathrm{~cm}$ for males $\geq 80 \mathrm{~cm}$ for female waist circumference increased from 42(10.6\%) to 48 (12.1) ( $p>0.05)$. The mean weight $57.7(S D \pm 10.5)$ at admission decreased to $57.4(S D \pm 10.6)$ at discharge $(P<0,000)$. Female in Sex, age $>60$ years old, rural in residence and anemia were factors associated underweight/under nourished before admission. Female in sex, age $>60$ years old, rural in residence and emergency type of current surgery, length of hospital stay $>15$ and anemia were factors associated underweight/under nourished.

Malnutrition is a frequent concomitant of surgical illness. A study reported that up to $40 \%$ of patients were malnourished at the time of their admission and majority of these patients continued to be nutritionally depleted throughout their hospital course (18). Similarly, in the current study the magnitude of under nutrient increased from 21.9-24.9\%. A similar unintentional weight loss was reported in a study conducted in Netherlands (19). In the current study, there is also significant mean weight depletion after the patients admitted to hospital. A significant weight loss was reported three months before admission in Ethiopian study (9).

The possible reasons for the continued under nutrition and weigh loss might be due diseases specific factors like loss of appetite, inflammation, swallowing difficulties, hyper catabolism; treatment related 
factors, such as episodes of fasting, side effects of treatment ; psychological factor (anxiety, depression, loneliness) or social factors (such as poverty) (1) and their clinical condition like fistula, malabsorption syndrome, intestinal obstruction and gastric atony $(20,21)$. The above condition can reduce dietary intake, absorption of macro- and/or micronutrients, increased losses or altered requirements, increased energy expenditure (in specific disease processes) $(21,22)$. In the current study majority of the patient were admitted for digestive tract surgery. A similar report indicates patients who undergo gastrointestinal surgery are at risk of nutritional depletion from inadequate nutritional intake and the stress of surgery both preoperatively and postoperatively (21).

Malnutrition is associated with poor tolerance to treatment decreased quality of life, and increased health care costs (23). In the current study, there is the longer duration of under nourished patients and septicemia and wound infection are the commonest complications. Malnourished patients have delayed in wound healing and longer length of stay reported in India (7). All the complications developed in the current study seems infectious which might acquire from hospital environment possibly drug resistance. Some studies had shown that infections are a classical complication of malnutrition and under feeding $(24,25)$.

The magnitude under nutrition before admission was $21.9 \%$. This was similar to study conducted from Brazil (22.2\%) (26) and China (17.8\%) (27). This was higher than another study conducted in Brazil (14.1\%) (3). However, it is lower than similar study from Ethiopia (55.6\%) (9) and pre-operative patients report from Albania (65.3\%) (2). The magnitude under nutrition in the current study increased to $24.9 \%$ during discharge This difference might be due to difference in socio demographic/ economic status ,sample size dietary habits, dietary diversity, types cases (malignant vs nonmalignant ), severity of illness (mild vs severely ill patients), types of surgery (elective vs emergency), hospital set up ,presence nutritional policy/support at hospital ,awareness of health professional about hospital malnutrition, nutritional assessment tool and support.

Despite the high prevalence of malnutrition, nutritional therapy was seldom prescribed to patients, thus reinforcing the fact that nutrition-related issues are not part of the patients' routine care in the current study. This increase in under nutrition and length of hospital stay after admission which can be possibly due to low awareness on hospital malnutrition among medical staff, low attention to given nutritional support medical staff, less application nutrition course given at 2 nd year of medical students training in our country university curriculum. In the current study setting, there is no weight and height scale which might simply use their physical judgment for nutritional assessment.

Routine evaluation of nutritional status was proposed which allows the identification of patients at risk of complications, particularly in the postoperative setting $(28,29)$. These patients should be targeted for specific nutritional support (30). However, only $16.8 \%$ of those with elective surgery before two weeks of admission and $43.5 \%$ of those who did surgery obtained nutritional support/counseling from the health professional. There are no official guidelines concerning hospital nutrition and the practice of nutritional therapy in the current study setting which can worsen the condition. 
Different factors were identified to be associated with under nutrition. The magnitude malnutrition was significantly higher in the age group $>60$ years. Similar significant report of malnutrition were reported among old age/elderly in studies from Albania (2), Spain (10), Taiwan (31), multi centered study from several Latin American countries (32) and Brazil $(26,3)$. That elder patient might have different condition which can impede or restrict nutritional intake like loose of appetites, co morbidities with chronic diseases. Diabetes mellitus and hyperextension are some of the chronic condition which requires nutritional restriction. Other reasons might be loneliness, depression, poor dentition and impairment of cognitive function, or secondary systemic disease. The magnitude under nutrition continues to increase from $47.1-52.1 \%$ among those with age greatest than 60 after surgery in this study. There is a need for nutritional support to elderly patients.

In the current study, males were less likely to undernourished. This is similar to study conducted from Brazil (33). The current study cannot establish cause-effect relationship because it's cross-sectional nature. However, this fact might be due to the physiology of female aging and the decline of anthropometric variables with age is also considerably higher in women than in men.

Length hospital stay was identified associated with increased under nutrition in the current study. This was similar to studies conducted from Brazil $(3,33)$, Australia (22) and multi centered study from Latin America (32).

Reducing length of hospital stay (LOS) has the potential to decrease health care cost, risk of infections and other hospital acquired diseases, and to improve patients' quality of life. Prolonged hospital stay may predispose a patient to skin colonization with more virulent hospital-based pathogens. Hospitals with reduced LOS are said to have done better than others with longer stay $(22,34,35)$. Reductions in postoperative length of stay may produce cost savings that can be invested in other areas of surgical patient care (36).

Those patients with emergency surgery are possibly under nourished than elective surgery in the current study. However, malnutrition was not related to the type of hospital admission (emergency or elective) or to the gender in other study (2).

This study used some basic nutritional assessment tool and biochemical assessment (hemoglobin). However, it did not include subjective assessment tool and other biochemical tests. This might provide additional information about the magnitude of the problems in the studied hospital.

\section{Conclusion}

In the current study the malnutrition /under nutrition is highly prevalent among surgical patients. Sex, age, residence and anemia were common factors associated with under nutrition at admission and discharge. In addition, type of current surgery and length of hospital stay were identified factors at time discharge. About one fifth and less than half percentage of patients obtained nutritional support before admission and at discharge. Therefore, the hospital staff should apply nutritional assessment and nutritional 
counseling /support to surgically admitted patients considering the identified factors. The hospital in which study conducted or the regional health bureau should also reinforce nutritional assessment and nutritional counseling /support/therapy through guideline development, training and monitoring its application. The regional health bureau should also work on implementable strategies considering the identified factors. Further studies should be conducted by using different nutritional assessment tool (subjective and objective), biochemical assessment and unidentified factors on different type's patients in the country. In addition, there is need for assessment on level nutritional counseling and factors associated with it among health professional working in the hospital.

\section{List Of Abbreviations}

AOR

Adjusted Odds Ratio; BMI:Body Mass Index; Cl:Confidence Interval; g/dl:Gram per Deciliter; IHRERC:Institutional Health Research Ethics Review Committee; IDF:International Diabetes Foundation; Kg:Kilogram; kg/m²:Kilogram per Millimeter Cube; LOS:Length of Stay; SPSS:Statistical Package for Social Sciences; WC:Waist Circumference; WHO:World Health Organization.

\section{Declarations}

\section{Ethics approval and consent to participate}

Ethical clearance for the study was obtained from the Institutional Health Research Ethics Review Committee (IHRERC) of Haramaya University. Letter support was written to Harari Regional Health Bureau from College of Health and Medical Sciences, Haramaya University. All methods were performed in accordance with the relevant guidelines and regulations. The objectives, risk and benefits of the study was explained to head of each hospitals and study participants. Written and signed informed consent were obtained from each study participants. The study participants' clinical records were reviewed anonymous. Information obtained during the study was kept confidential and only intended for research purpose.

\section{Consent for publication}

This manuscript had not published and not submitted to another journal. We hereby transfer(s), assign(s), or otherwise convey(s) all copyright ownership, including any and all rights incidental there to, exclusively to BMC Nutrition in the event that such work is published by BMC Nutrition owns the work including: copyright, the right to grant permission to republish the article in whole or in part, with or without fee, the right to copy, distributes, and displays the work, the right to produce preprints or reprints and translate in to language other than English for sale or free distribution; and the right to republish the work in a collection of articles in any other mechanical or electronic format.

We give the rights to the corresponding author to make necessary changes as per the request of the journal, do the rest of the correspondence on our behalf and he will act as the guarantor for the 
manuscript on our behalf.

\section{Competing interests}

All the authors declare that they have no competing of interest associated with the publication of this manuscript.

\section{Funding}

No funding

\section{Availability of data and materials}

The authors declare that all the necessary data are fully described within the manuscript.

\section{Authors' contributions}

Zelalem Teklemariam participated in proposal writing, data collection, analysis, interpretation and critical review of the manuscript. Fitsum Weldegebreal participated in proposal writing, data collection and critical review of the manuscript. Habtamu Mitiku participated in proposal writing, data collection, analysis and critical review of the manuscript. All authors read and approved the final manuscript.

\section{Acknowledgement}

We would like acknowledge all study participants for their unreserved support in providing information and data collector for their endurance during data collection process. We would also to express our deepest gratitude to Institutional Health Research Ethics Review Committee (IHRERC) of Haramaya University for facilitation ethical clearance process and Haramaya University for providing fund to conduct this research.

\section{References}

1. Anonymous. Group DMS Recognition and treatment of undernutrition in hospital inpatients and outpatients. Amsterdam: vrijeuniversiteit. 2015.

2. Shpata V, Prendushi X, Kreka M, Kola I, Kurti F etal. Malnutrition at the Time of Surgery affects negatively the Clinical Outcome of Critically III Patients with Gastrointestinal Cancer in university of Hospital of Albania. 2014; 68:263-7.

3. Leandro-Merhi VA, Aquino jlbd, Chagas JFS. Nutrition Status and Risk Factors Associated with Length of Hospital Stay for Surgical Patients School of Medicine, Puc-Campinas-SP, Brazil. Journal of Parenteral and Enteral Nutrition. 2010;35(2):241-8.

4. Saunders J, Smith T. Malnutrition: causes and consequences Southampton University Hospitals, Southampton. CME Nutrition 2010; 10(6):624-7. 
5. Edington J,Boorman J,Durrant ER, Perkins A, Giffın CV, James R, Thomson JM, Oldroyd JC, Smith JC, Torrance AD, Blackshaw V, Green S, Hill CJ, Berry C, McKenzie C, Vicca N, Ward JE, Coles SJ. 2000. Prevalence of malnutrition on admission to four hospitals in England. ClinNutr 19(3):191-5.

6. Pirlich M, Schütz T, Norman K, Gastell S, Lübke HJ, Bischoff SC, Bolder U, Frieling T, Güldenzoph H, Hahn K, Jauch K-W, Schindler K, Stein J, Volkert D, Weimann A, Werner H, Wolf C, Zürcher G, Bauer P, Lochs H. The German hospital malnutritionstudy. ClinNutr.2006;25(4):56372.

7. Mahakalkar CC, Modi S, Yeola M, Kaple MN, Patwardhan MA et al. Malnutrition in hospitalized patients; a real concern in surgical outcomes Maharashtra India International Journal of Research in Medical Sciences. 2013; 2(1):250-7.

8. Meijers JM,Schols JM,van Bokhorst-de van der Schueren MA, Dassen T, Janssen MA, Halfens RJ. Malnutrition prevalence in The Netherlands: results of the Annual Dutch National Prevalence Measurement of Care Problems Brit J Nutr. 2009; 101(3): 417-23

9. Haile A, Hailu M, Tesfaye E. Prevalence and associated factors of malnutrition among adult hospitalized patients at Amhara National Regional State Referral Hospitals, Ethiopia. Integrative Obesity and Diabetes. 2015;1(3):80-3.

10. Álvarez-Hernandez J, Vila MP, León-Sanz M. Prevalence and costs of malnutrition in hospitalized patients Barcelona. España the PREDyCES® Study. Nutrition Hospitalcia 2012; 27(4):1049-59.

11. Garcia RS, Tavares LRdC, Pastore CA. Nutritional screening in surgical patients of a teaching hospital from Southern Brazil: the impact of nutritional risk in clinical outcomes. Einstein. 2013; 11(2):147-52.

12. Correia ML, Caiaffa WT, da Silva AL, Waitzberg DL. Risk factors for malnutrition in patients undergoing gastroenterological and hernia surgery in University of São Paulo Medical School, Brazil: an analysis of 374 patients. Nutr Hosp. 2001;16(2):59-64.

13. FAO. Nutrition and Consumer Protection Division, Rome 2007.

14. World Health Organization (WHO). Obesity: Preventing and Managing the Global Epidemic: Report of a WHO Consultation on Obesity. Geneva, Switzerland. 1998.

15. Lipschitz DA. Screening for nutritional status in the elderly.Prim Care. 1994; 22: 55-67.

16. International Diabetes Federation. Worldwide definition of the metabolic syndrome. Available at: 2005. http://www.idf.org/metabolic_syndrome.

17. WHO. Haemoglobin concentrations for the diagnosis of anaemia and assessment of severity. Vitamin and Mineral Nutrition Information System. Geneva, World Health Organization, 2011 (WHO/NMH/NHD/MNM/11.1) (http://www.who.int/vmnis/indicators/haemoglobin. pdf, accessed 7/11/2016).

18. Beattie AH, Prach AT, Baxter JP, Pennington CR. A randomized controlled trial evaluating the use of enteral nutritional supplements postoperatively in malnourished surgical patients. Gut. 2000; 46: 813-818.

19. Loh KW, Vriens MR, Gerritsen A, Borel Rinkes IH, van Hillegersberg R, et al. Unintentional weight loss is the most important indicator of malnutrition among surgical cancer patients. Neth J Med 2012;70: 


\section{5-369.}

20. Senesse P, Assenat E, Schneider S, Chargari C, Magne N, AzriaD, Hebuterne X. Nutritional support during oncologic treatment of patients with gastrointestinal cancer: who could benefit?Cancer Treat Rev. 2008; 34: 568-575.

21. Mariette C, De Boton ML, Piessen G. Surgery in esophageal and gastric cancer patients: what is the role for nutrition support in your daily practice? Ann Surg Oncol. 2012; 19: 2128-2134

22. Laky B, Janda M, Kondalsamy-Chennakesavan S, Cleghorn G, Obermair A.2010. malnutrition and quality of life association with prolonged length of hospital stay among patients with gynecological cancer in Australia. BioMedcenteral 10:232-8.

23. van Bokhorst-de van der Schueren MA. Nutritional support strategies for malnourished cancer patients. Eur J Oncol Nurs.2005; 9(Suppl 2): S74-83.

24. Villet S, Chiolero R, Bollmann M, Revelly J, Cayeux M, DelarueJ, et al. Negative impact of hypocaloric feeding and energy balance on clinical outcome in ICU patients. Clin Nutr. 2005; 24(4): 502-509.

25. Rubinson L, Diette GB, Song X, Brower RG, Krishnan JA. Lowcaloric intake is associated with nosocomial bloodstream infections in patients in the medical intensive care unit. Crit Care Med. 2004; 32: 350-357.

26. Valente da Silva H.G., Santos S.O., Silva N.O., Ribeiro F.D., Josua L.L. and A. S. B. Moreira A.S.B. Silva HGVd, S. O. Santos NOS, F. D. Ribeiro LLJ, Moreira ASB. 2012. Nutritional assessment associated with length of inpatients' hospital stay Rio de Janeiro State University Brazil. Nutrition Hospitalcia. 27(2):542-7.

27. Fang S, Long J, Tan R, Mai H, Lu W, et al. (2013) A multicentre assessment of malnutrition, nutritional risk, and application of nutritional support among hospitalized patients in Guangzhou hospitals. Asia Pac J Clin Nutr 22: 54-59.

28. Van Cutsem E, Arends J. The causes and consequences of cancer-associated malnutrition. Eur J Oncol Nurs. 2005; 9 suppl 2: S51-63.

29. Kondrup J, Allison SP, Elia M, Vellas B, Plauth M. ESPEN Guidelines for Nutrition Screening 2002. Clinical Nutrition.2003; 22(4): 415-421.

30. Weimann A, Braga M, Harsanyi L, et al. ESPEN guidelines onenteral nutrition: surgery including organ transplantation. ClinNutr. 2006; 25: 224-244.

31. Huang th, chi cc, liuch, chang c, kuo Im etal.2014. Nutritional Status Assessed by Scored Patient generated Subjective Global Assessment Associated with Length of Hospital Stay in Adult Patients Receiving an Appendectomy. Biomed J 37:71-7.

32. Correia M, Campos A.2003. Prevalence of hospital malnutrition in Latin America. The multicenter ELAN study Nutrition. 19(10):823-5

33. Barbosa LRLS, LacerdaFilho A, Barbosa LCLS.2014. Immediate preoperative nutritional status of patients with colorectal cancer: a warning Brazil ArqGastroenterol 51(4):331-6. 
34. Ilesanmi OS, FatiregunAA. 2014. Length of Stay of Surgical Inpatients at University College Hospital, Ibadan, Nigeria. British Journal of Medicine \& Medical Research. 4(10):1992-2001.

35. Sang SLW, Chaturvedi R, Alam A, Samoukovic G, Varennes Bd, Lachapelle K. 2013.Preoperative hospital length of stay as a modifiable risk factor for mediastinitis after cardiac surgery in Canada. Journal of Cardiothoracic Surgery. 8(45):1-7.

36. Gagarine A, Urschel JD, Miller JD, Bennett WF, Young JEM.2003. Preoperative and Intraoperative Factors Predictive of Length of Hospital Stay after Pulmonary Lobectomy in Boston USA. Ann Thorac Cardiovascular Surg. 9(4):222-5. 